Published in final edited form as:

Neuropsychol Dev Cogn B Aging Neuropsychol Cogn. 2011 November ; 18(6): 653-677. doi: 10.1080/13825585.2011.613450.

\title{
Space Fortress game training and executive control in older adults: A pilot intervention
}

\author{
Yaakov Stern ${ }^{1}$, Helena M. Blumen ${ }^{1}$, Leigh W. Rich ${ }^{1}$, Alexis Richards ${ }^{1}$, Gray Herzberg ${ }^{1}$, and \\ Daniel Gopher ${ }^{2}$ \\ ${ }^{1}$ Taub Institute, Columbia University, Cognitive Neuroscience Division, New York, NY, USA \\ ${ }^{2}$ Industrial Engineering and Management, Technion - Israel Institute of Technology, Haifa, Israel
}

\section{Abstract}

We investigated the feasibility of using the Space Fortress (SF) game, a complex video game originally developed to study complex skill acquisition in young adults, to improve executive control processes in cognitively healthy older adults. The study protocol consisted of 36 one-hour game play sessions over 3 months with cognitive evaluations before and after, and a follow-up evaluation at 6 months. Sixty participants were randomized to one of three conditions: Emphasis Change (EC) - elders were instructed to concentrate on playing the entire game but place particular emphasis on a specific aspect of game play in each particular game; Active Control (AC) - game play with standard instructions; Passive Control (PC) - evaluation sessions without game play. Primary outcome measures were obtained from five tasks, presumably tapping executive control processes. A total of 54 older adults completed the study protocol. One measure of executive control, WAIS-III letter-number sequencing, showed improvement in performance from pre- to post-evaluations in the EC condition, but not in the other two conditions. These initial findings are modest but encouraging. Future SF interventions need to carefully consider increasing the duration and or the intensity of the intervention by providing at-home game training, reducing the motor demands of the game, and selecting appropriate outcome measures.

\section{Keywords}

Aging; Cognition; Intervention; Executive control; Video games

\begin{abstract}
Epidemiological evidence suggests that a set of lifetime exposures including educational and occupational attainment and leisure activities later in life are associated with more preserved cognitive and day-to-day function in aging, and with reduced risk of incident dementia. However, the specific set of activities that can maintain or improve function in late life has not yet been defined. A great deal of recent research has focused on developing cognitive interventions that may compensate for age-related cognitive decline (see Green \& Bavelier, 2008; Hertzog, Kramer, Wilson, \& Lindenberger, 2008; Lustig, Shah, Seidler, \& ReuterLorenz, 2009; Noack, Lövdén, Schmiedek, \& Lindenberger, 2009; Papp, Walsh, \& Snyder, 2009; Schmiedek, Bauer, Lövdén, Bose, \& Lindenberger, 2010 for reviews). A major problem that has plagued most cognitive intervention trials in cognitively healthy older adults is that training in one cognitive domain does not reliably generalize to other cognitive domains, or to day-to-day activities. For example, the Advanced Cognitive Training for Independent and Vital Elderly (ACTIVE) study found that training within a cognitive
\end{abstract}

C 2011 Psychology Press, an imprint of the Taylor \& Francis Group, an Informa business

Address correspondence to: Yaakov Stern PhD, Columbia University, P\&S Box 16, $630 \mathrm{~W} 168^{\text {th }}$ Street, New York, NY 10032, USA. ys11@ columbia.edu. 
domain persisted over time, but there was no transfer of training across cognitive domains (Willis et al., 2006). Many cognitive intervention trials in cognitively healthy older adults also fail to demonstrate transfer of training to different tasks within the same cognitive domain. For example, a meta-analysis of 33 studies of cognitively healthy older adults trained in the use of some mnemonic technique (such as the method of loci or imagery) reported that memory improvements were for the most part specific to memory tasks that lent themselves to the previously trained mnemonic technique (Verhaeghen, Marcoen, \& Goossens, 1992; see also Rebok, Carlson, \& Langbaurn, 2007).

Cognitive interventions specifically aimed at training executive control have been more effective in demonstrating transfer of training to other tasks within the same cognitive domain (Bherer et al., 2005, 2008; Karbach \& Kray, 2009; Kramer, Larish, \& Strayer, 1995), and day-to-day activities (Willis et al., 2006). Executive control processes presumably monitor and coordinate complex behaviors such as planning, abstract thinking, reasoning, and the selection and inhibition of appropriate actions (Norman \& Shallice, 1986). There is also good evidence that training within the context of complex computer games may be particularly useful for enhancing executive control. For example, one recent study, suggest that computer-based training with an off-the-shelf strategy game called Rise of Nations show transfer of training to a range of different executive control tasks (Basak, Boot, Voss, \& Kramer, 2008). This could be because computer-based training tasks can be structured to have an inherent complexity that places a high demand on executive control. In addition, they lend themselves to training programs that extend over weeks or months, which may also be important for developing more enduring and stable capacities that can transfer to performance on other executive control tasks (Basak et al., 2008; Drew \& Waters, 1986; Frederiksen \& White, 1989; Gopher, Weil, \& Siegel, 1989; Gopher, Weil, \& Bareket, 1994; Green \& Bavelier, 2003, 2006, 2007).

The cognitive reserve hypothesis suggests that certain aspects of life experiences, strongly associated with measures of educational attainment, occupational attainment, and leisure activities, contribute to a person's ability to cope with or compensate for age-related brain changes in order to maintain cognitive task performance and day-to-day activities (see Stern, 2009 for a recent review). One important feature of cognitive reserve is that it is not taskspecific, but should provide a buffer for brain changes across multiple sets of cognitive tasks, cognitive domains, and day-to-day activities. From a cognitive intervention point of view, therefore, it would be important to define the specific set of activities that can increase cognitive reserve.

Two recent studies suggest that there is a relationship between cognitive reserve and executive control. In an fMRI study designed to identify a 'cognitive reserve network', we identified an activation pattern that increased with task demand across two separate tasks and, more importantly, was utilized to a greater degree by individuals with greater cognitive reserve (Stern et al., 2008). Notably, a major component of this activation pattern was areas associated with executive control. In another study, large sets of cognitive task performance was analyzed with structural equation modeling in order to determine whether proxies of cognitive reserve (such as Verbal IQ, Vocabulary, and Years of Education) formed a latent construct that was separate from other cognitive domains such as memory, speed of processing, and executive control (Siedlecki et al., 2009). This study not only found that cognitive reserve proxies formed a separate latent construct, but also that this construct was most strongly associated with that representing executive control. Taken together, these studies provide strong support for an association between cognitive reserve and executive control. 
In the current study, we investigated the feasibility of using the Space Fortress (SF) game, a complex video game originally developed to study complex skill acquisition in young adults, to improve executive control in cognitively healthy older adults. The SF game was originally developed to study complex skill acquisition in young adults. In the SF game, the player is required to shoot missiles and destroy a space fortress while protecting their spaceship against damage. The spaceship and the missiles are controlled with a joystick. In addition to defending the spaceship against missiles shot by the space fortress, the player has to defend themselves against two types of mines that intermittently appear on the screen. The player is also asked to monitor symbols that randomly appear on the screen. In the original research framework in which the SF game was developed (Mané \& Donchin, 1989), elements and components of the game were specifically developed to represent divided attention, multi-tasking, visual scanning, working memory and long-term memory load, dynamic and discrete motor control. An individual's ability to control or switch attention to different task requirements is considered vital for successful SF game performance (Arthur et al., 1995; Gopher, 2006; Gopher et al., 1989, 1994), and therefore training on this task is expected to enhance executive control. Unlike off-the-shelf computer-based games such as Rise of Nations (Basak et al., 2008), the SF game can be customized to compute sub-scores that reflect these different task components.

In contrast to off-the-shelf computer-based games, the SF game also allowed us to customize and test a training approach specifically designed to enhance executive control: Emphasis Change (EC) training (Gopher et al., 1989). In the EC training approach, participants are instructed to play the entire game at all times, but to shift their emphasis to different subcomponents of the game at different times during training. In the context of the SF game, the EC training approach encourages participants to attempt to play the entire game, but at different times place more attention on either controlling the spaceship or handling mines. This EC training approach has been shown to be superior to ad lib game play in numerous studies of younger adults across multiple tasks including SF (Fabiani et al., 1989; Gopher, 2007; Gopher et al., 1989, 1994) and in one study of older adults using another executive control task (Kramer et al., 1995). EC instructions encourage individuals to explore alternate learning strategies, and increase the demand for executive control (Gopher, 2007; Gopher et al., 1989, 1994; Kramer et al., 1995).

In one large-scale study of young adults, EC and a second training paradigm (training by parts) emerged as the most successful in enhancing overall SF performance across 10 training sessions (Fabiani et al., 1989). Training by parts involved successively learning to play the SF game by practicing tasks that engage only one, or a few different SF task requirements. When these two strategies were compared directly, the training by parts approach yielded higher overall SF performance at the end of training. However, subsequent to training a secondary task was introduced to be performed along with SF and individuals trained with EC instructions were better able to incorporate the secondary task. Gopher et al. (1989) have suggested that the EC training approach enhances the 'task shell' that coordinates performance on all of the various task components. Therefore this training approach facilitates incorporating additional tasks.

In younger adults, several studies have also shown that the EC training approach to the SF game produces effective transfer of training to real-life activities. For example, EC training of the SF game was associated with better flight simulation and flying performance in Israel Air Force candidates, and with improved training in US Army helicopter pilots (Gopher et al., 1994). We therefore hypothesized that SF training using the EC approach might enhance executive control processes in elders in a way that could generalize to other executive control tasks (Fabiani et al., 1989; Frederiksen \& White, 1989; Gopher et al., 1994; Green \& Bavelier, 2003, 2006, 2007; Kramer et al., 1995), and real-life activities - although this 
initial study focused on whether the EC training approach to the SF game would transfer or improve performance on other executive control tasks.

More specifically, in the current study, healthy, cognitively normal elders were randomized into three conditions. The EC condition played space fortress with EC instructions. A second, Active Control (AC) condition played the game, but with no EC training. A third Passive Control (PC) condition underwent pre-and post-evaluations but did not engage in any game play. Because SF had never been played by older participants, we modified procedures that had been used in studies of younger adults. A standard session of SF game play consists of 12, 3-minute games. In previous studies of younger adults, the typical experiment consisted of 10 training sessions. Because of the difficulty of the game, and to enhance its potential to influence performance on other tasks, we instituted a longer training period consisting of 36 sessions conducted over 12 weeks. Because elders are typically unfamiliar with computer games and the joystick, we provided detailed guidance and feedback about game play to all participants in the first several sessions. We also altered the typical criteria that have been applied to screen potential participants for motor skill. When playing the SF game, the dynamic control and aiming of the ship is an exceptionally difficult task that involves precise, discrete, and continuous motor control. In studies of younger adults, potential participants were screened with an aiming task to ensure that they had sufficient motor skills to participate in the study. The aiming task is based upon the aiming component of the SF game and involves aiming and shooting missiles with a stationary space ship. Typically, an aiming score less than 780 have been used as the criteria for exclusion (e.g., Gopher et al., 1989; Mané \& Donchin, 1989). In the current study of cognitively healthy older adults we did not exclude participants with low aiming scores because this would likely lead to the exclusion of a large amount of participants and would counteract our desire to begin developing an intervention that might help elders compensate for age-related cognitive decline.

No study had ever examined SF game play in elderly participants so we were very interested in whether the elders would tolerate playing this difficult game, maintain participation over the 36 sessions, and show improvement in their game play. These questions have been addressed in a separate paper which focuses on how elders in the EC and AC conditions learn to play the game and on potential differences in learning between the two conditions (Blumen, Gopher, Steinerman, \& Stern, 2010). In brief, while they found the game to be very difficult, the older adults did show improvement in SF performance over time, but did not perform at the same level as younger adults. As will be described later, over the training period the AC condition showed a greater improvement than the EC condition in overall or Total SF performance. This is in contrast to studies in young adults where the opposite result has consistently been noted (e.g., Gopher et al., 1989). Note that overall or Total SF performance reflects four different task components or sub-scores: Points (which increases from shooting and destroying the fortress and mines, and from monitoring symbols that regularly popped up on the screen), Velocity (which increases from flying the ship slowly and decreases from flying the ship fast), Control (which increases from keeping the ship within the hexagon and decreases from flying outside of the hexagon), and Speed (which is proportional to the efficiency of detecting and destroying mines). Thus, while some subscores add to the Total other sub-scores subtract from the Total, hence, allowing for both positive and negative overall SF performance. However, we also noted that game play was qualitatively different for participants in the EC vs. the AC condition. More specifically, the overall advantage in SF performance for the AC condition was primarily due to collecting more bonus points from monitoring symbols that regularly popped up on the screen and not - the primary goal of the game - shooting and destroying fortress, which in contrast benefited from EC instructions. We therefore wanted to determine whether there was differential benefit from game play under these two task conditions as well. Of course both 
game play conditions provided extended experience playing a very challenging game that required executive control, so we also entertained the possibility that elders engaged in game play in general would show greater improvement on our outcome measures than those in the $\mathrm{PC}$ condition.

Our primary outcome measures were selected measures of executive control because we hypothesized that SF game play, particularly when played in the EC condition, would provide a specific benefit to cognitive functions assessed by the executive control tasks. In order to provide a more complete cognitive profile of study participants at baseline, and throughout the course of this intervention, we also included a comprehensive battery of tests assessing multiple cognitive domains including memory, speeded processing, language, and spatial ability - but did not develop any formal hypotheses for these measures. The inclusion of a comprehensive battery of cognitive assessments is also important for testing our hypothesis that SF game play with the EC training approach will improve executive control processes in particular and not overall cognitive functions in general. Finally, in addition to assessing cognitive function prior to and immediately after the 12-week training period, we included an additional assessment 3 months later to assess the potential long-term benefit of game play.

\section{METHODS}

\section{Participants Inclusion Exclusion Criteria}

Cognitively healthy older adult participants were recruited via fliers, internet ads, and visits to local senior centers. Participants were fluent in English, had normal or corrected-tonormal vision and reported no past or current medical, neurological or psychiatric disorder. Participants were screened for dementia with the Mattis Dementia Rating Scale (DRS; Mattis, 1973/1988). A total DRS score of 135 or higher were required for inclusion. In addition, neuropsychological test data from the baseline assessment battery were reviewed to ensure the absence of dementia or MCI.

\section{Procedure}

Overview-After signing informed consent, all participants were screened with the DRS in order to rule out the possibility of dementia. All participants then completed a baseline assessment battery, which consisted of paper-and-pencil neuropsychological instruments and computerized cognitive instruments. An aiming task, originally developed as a motor abilities screen for the SF game, was also a part of this baseline assessment battery. The computerized cognitive instruments were re-administered to all participants after 6 weeks (i.e., the midpoint of the training period). The full assessment battery was re-administered at the end of the training period (i.e., at 12 weeks) and then again 3 months following the end of the training period. Twenty participants were randomly assigned to each of three conditions: EC, AC, and PC. The EC condition played the SF game three times a week for 12 weeks with an EC training protocol. The AC condition played the SF game for 12 weeks with a standard SF training protocol. The PC condition received the full assessment battery at the same times as the other two conditions, but did not participate in SF game training.

\section{Assessment Battery}

Aiming Task-All participants completed three trials of the aiming task that is based upon the aiming component of the SF game. During the aiming task, the player learns to aim and shoot missiles with a stationary spaceship. A total aiming score is obtained by adding the number of successful missiles shot within a given trial (points) and the speed of missiles shot with a given trial (speed). 
Neuropsychological Assessments-The neuropsychological battery included assessments of Speed: WAIS-III Digit Symbol subtest (Wechsler, 1997) and Trail Making Test Part A (Reitan, 1978), Visuoconstruction: WAIS-III Block Design subtest, Memory: Total Recall on the California Verbal Learning Test: Second Edition (CVLT-II total; Delis, Kramer, Kaplan, \& Ober, 2000), Language: Controlled Oral Word Association Test (Benton \& Hamsher, 1976) Category Fluency Test (Goodglass \& Kaplan, 1983), and Executive Functions: WAIS-III Letter-Number Sequencing (Wechsler, 1997) Stroop Color and Word Test (Golden, 1978) and the Trail Making Test Part B (Reitan, 1978).

Computerized Cognitive Assessments-The computerized cognitive battery included two different tasks: a set-switching task and a flanker task.

Set-switching Task-This task was composed of two different tasks: a letter classification task and a digit classification task. In the letter classification task, participants were presented with a letter and indicated via differential button press whether it was a consonant or a vowel. In the digit classification task, participants indicated whether a presented digit was odd or even. Each experimental trial began with a 200-ms fixation symbol at the center of the screen followed by a $100-\mathrm{ms}$ delay and then a digit or a letter. Participants were instructed to respond as quickly and as accurately as possible and their response initiated the next trial. The experimental session was divided into eight blocks of 40 trials. During four of these blocks, participants performed only the letter or the digit task. During the four other blocks, the tasks shifted within the block. Participants were informed about the nature of the block at the beginning of each block. The nature of the task was also specified by shifting background color (blue, letter classification; green, digit classification). The primary dependent measures on this task were the difference in RT between switch and non switch trials within a block, i.e., local switch costs and the difference in RT between switch and non switch blocks, i.e., global switch costs (Karbach \& Kray, 2009; Reimers \& Maylor, 2005).

Flanker Task-In this task, participants were presented with a line of 5 arrows that point either to the right $(>)$ or left $(<)$ and were asked to identify the orientation of the central arrow, while ignoring the orientation of the peripheral arrows. On 50\% of the trials, the peripheral arrows pointed in the same direction as the central arrow (e.g., $\ll \ll<<)$ - i.e., compatible distracters. On the other $50 \%$ of the trials, the peripheral arrows pointed in the opposite direction as the central arrow (e.g., $\gg\langle\gg)$ ) - i.e., incompatible distracters. There was one 4-minute practice block and three 4-minute test blocks of trials with an equal number of randomly distributed compatible and incompatible distracters. The stimuli were presented for 2 seconds on each trial followed by a randomly jittered 4-8-second ISI. The ISI was jittered to optimize statistical power in future imaging studies using this task (Smith, Jenkinson, Beckmann, Miller, \& Woolrich, 2007). Thus, participants performed a total of 120 experimental trials. The primary dependent measure was the difference in reaction time for incompatible vs. compatible distracters, i.e., flanker interference.

Randomization-Participants were randomly assigned to one of the three study conditions (EC, AC, or PC). The randomization was blocked so that each set of 20 participants were distributed equally across the three conditions. Participants were not informed about the differences between AC and EC conditions and therefore could be considered blinded to condition. Examiners were aware of the study condition assignments because of training requirements associated with these two conditions. 


\section{Apparatus}

Space Fortress Task-The revised version of the SF game (Shebilske et al., 2005) was installed on three networked PC computers. The primary goal of this game was to shoot missiles and destroy a space fortress while protecting your spaceship against damage. The spaceship and the missiles were controlled by the player with a joystick (Flightstick Pro) and the spaceship moved in a frictionless environment. Any forward movement of the joystick made the spaceship accelerate and any lateral movement of the joystick made the spaceship rotate. If the ship was steered off the screen, it would reappear (or wrap) to the other side of the screen. If a button on top of the joystick was pressed, a missile was fired in the direction of the spaceship. In addition to defending the spaceship against missiles shot by the space fortress, the player had to defend themselves against mines that appeared on the screen every 4 seconds. There were two types of mines: friend mines and foe mines. When friend mines were shot they were energized, ran into the space fortress and scored a hit. When foe mines appeared, the player had to switch the weapon system by pressing the right mouse button twice and then eliminate the mine with a missile. Prior to each trial, the player was told what three letters signified foe mines, which they then compared to a single letter displayed on the bottom of the screen whenever a mine appeared. A different set of three letters signified foe mines during each trial. The space fortress had to be shot 10 times with more than $250 \mathrm{~ms}$ between each shot before it became vulnerable, then the space fortress could be destroyed with a double shot (i.e., two shots against the space fortress administered with no more than $250 \mathrm{~ms}$ between each shot). Bonus points could also be obtained by monitoring symbols that regularly popped up on the screen. Whenever the player saw $2 \$$ signs in a row, she could press the left mouse button and choose to receive bonus points or additional missiles.

Space Fortress Game Training-Our training program in general and our implementation of EC in particular, differed from previous SF game learning studies of younger adults. Typically, training in younger adults has consisted of 10 one-hour training sessions spaced over a couple of weeks (e.g., Gopher et al., 1989). We extended our training in older adults to 3 one-hour sessions weekly for 12 weeks (i.e., a total of 36 one-hour sessions). Following standard game play instructions, players are assigned to the EC condition are instructed to focus on ship handling on some trials, mine handling on other trials or both ship handling and mine handling on some trials (Gopher et al., 1989). We also took additional measures to ensure that the older adults would stick with the 3-month training program. During the first training session, each participant watched a flash tutorial about the SF game. The standard instructions were then read to them, allowing for questions along the way. Participants in the EC condition were also told to focus on flying the spaceship slowly and between the hexagons and maximize their Control and Velocity subscore. During the first week of training, the experimenter also sat down with each participant (regardless of whether assigned to the EC or AC condition) and reviewed their performance after each game trial, not only in terms of their Total score but also in terms of their subscores. Hence, both conditions were given EC instructions during the early training sessions because they were alerted to improve on different aspects of the game. However, on top of that, participants in the EC condition were told to focus on ship handling during some trials and on mine handling during other trials.

Participants completed a total of $36 \mathrm{SF}$ training sessions over a 3-month period. Each training session consisted of 12 three-minute game trials. Participants in the AC condition completed three training sessions, three times a week with standard game instructions (i.e., participants were told to maximize their Total game score, and use any strategy they felt would help them succeed). Participants in the EC condition also completed three training sessions three times a week for 12 weeks. However, during the first 10 trials in each session, participants were told to either focus on ship handling (i.e., participants were told to focus 
on flying the spaceship slowly and between the hexagons and maximize their Control and Velocity sub-score) or on mine handling (i.e., participants were told to focus on dealing with friend and foe mines and maximize their Speed sub-score). During the last 2 trials of each training session, participants in the EC condition also played the game with standard game instructions. Our implementation of EC instructions across training sessions can be summarized as follows. During the first 4 weeks of training, EC participants were asked to focus solely on ship handling. During weeks 4-6, EC participants were asked to focus on mine handling. Finally, during weeks $7-12$ ship handling and mine handling were intermixed within sessions and trials. Note that during the 11th and the 12th trial in each session, both EC participants and AC participants received standard game instructions.

\section{Statistical Approach}

Our main set of analyses focused on change in performance from base-line to the end of the 3-month training period. For the neuropsychological instruments this consisted of two assessments: baseline (pre) and 3 months (post). For the computerized cognitive instruments, this consisted of three assessments: pre, 6 weeks (mid), and post. Because our hypothesis was that SF game play with the EC training protocol would lead to improvement in executive control processes, our primary outcome measures were selected from the five tests in our assessment battery that could be used to assess this domain: the Trail Making Test, the Letter Number Sequencing Test, the Stroop Color and Word Test, the SetSwitching Task, and the Flanker Task. These measures included the time to complete Trails B corrected for Trails A from the Trail Making Test (Trails B-A), the raw score from the letter-number sequencing task WAIS-III, the raw response time to Color-Word (incongruent) trials corrected for Color (congruent) trials from the Stroop Color and Word Test (i.e., Stroop Interference), the local and global switch costs in response time from the Set Switching task, and the flanker interference response time measure from the Flankers task. Although five of these six measures are difference scores, our analyses were completed on raw scores. More specifically, for these measures we used repeated-measures analyses that included time of testing (pre and post, or pre, mid and post) and trial type as within subjects factors, and condition (EC, AC, and PC) as the between-subjects factor. Trial type for the Trail Making Test was Trails A and Trails B. Trial Type for the Stroop Color and Word Test was congruent trials and incongruent trials. Trial type for the Set-Switching Task was single (non-switch trials in non-switch blocks), repeat (non-switch trials in switch blocks) and switch (switch trials in switch blocks). Finally, trial type for the Flanker task was compatible and incompatible trials. Consideration of these analyses focused on the three-way interaction between time of test, trial type, and condition. For the letter-number sequencing task, and additional neuropsychological assessments (WAIS-III Digit Symbol subtest, WAIS-III Block Design Subtest, CVLT-II total, Controlled Oral Word Association Test and Category Fluency Test) we used repeated measure analyses that included time of testing (pre and post) as the within subjects factor and condition (EC, AC, and PC) as the between-subjects factor. Consideration of these analyses focused on the two-way interaction between time of test and condition. Analyses focused first on the primary outcome variables during training and to the end of training period, and subsequently on the remaining additional neuropsychological assessments and the 3-month follow-up testing.

\section{RESULTS}

\section{Participants}

A total of 87 potential participants were screened for this study, and 60 were randomized into the three study conditions. Twelve participants were excluded prior to inclusion because their total DRS score was less than 135, nine participants were excluded because they reported a past or current medical, neurological or psychiatric disorder, two participants 
were excluded because of language or learning disabilities, and three participants were excluded because they could not make the time commitment. Demographic and screening information for included participants in each condition are provided in Table 1. Note that participants in the three conditions did not differ in terms of Age, Percent Female, Years of Education or total DRS score $(t s<1)$. A total of 6 participants did not complete the study protocol: 3 from the $\mathrm{EC}$ condition, 2 from the $\mathrm{AC}$ condition, and 1 from the control condition. Two of these participants could not make the time commitment, one got a job, one lost interest at follow-up, one could not complete the training program due to arthritis in the arm and the neck, and one felt the study did not pay enough for his time.

\section{Space Fortress Performance}

Detailed analyses of how the AC and EC training conditions learned the SF game are presented in a separate paper (Blumen et al., 2010). Several aspects of game play potentially relevant to the cognitive transfer of game play are reviewed here. Recall that during the 11th and the 12th trial in each session, both EC participants and AC participants received standard game instructions. Analysis of game learning focuses on scores in these final two games played at each training session.

As illustrated in Figure 1, study participants showed improved performance on the SF task over time. However, unlike previous reports in younger adults, improvement on SF total score was greater in the AC condition than in the EC training condition (Gopher et al., 1989). This advantage was primarily due to participants in the AC condition collecting more bonus points (Figure 2). In contrast several measures associated with the primary goal of the game including shooting and destroying the fortress benefited from EC instructions (Figure $3)$. These observations suggest that learning and playing the game under the EC condition was a qualitatively different experience than playing the game under the $\mathrm{AC}$ condition. We also found that initial motor control abilities, as measured by the aiming task, influenced many different aspects of SF game learning and often interacted with learning rate and training instructions.

\section{Primary Outcome Measures}

Repeated measures analyses for the primary outcome measures are summarized in Table 2 . Means and standard deviations for primary outcome variables are shown in Table 3. There was a main effect of trial type on the Trail making test, $F(1,50)=273.60, p<.001$, and the Stroop Color and Word test, $F(1,50)=690.50, p<.00$. These main effects nicely replicate previous findings of slower response time to Trails B relative to Trails A on the Trail Making test and to incongruent relative to congruent trials on the Stroop Color and Word Test (i.e., Stroop Interference). There was also main effect of time of test and trial type on response time during the set-switching task, $F(2,96)=4.75 p<.05$ and $F(2,96)=58.47, p<$. 001 , respectively. ${ }^{1}$ Follow-up (Helmert) contrasts indicated that overall response time on the set-switching task improved from baseline to mid- and post-testing, $F(1,48)=6.50, p<.05$, but did not improve from mid- to post-testing $F(1,48)=1.85, p>.05$. Helmert contrasts also indicated that response times were faster during single (non-switch trials in non-switch blocks) than during repeat (non-switch trials in switch blocks) and switch trials (switch trials in switch blocks; i.e., global switch costs, $F(1,48)=34.35, p<.001$ ), and that response time was faster during repeat and switch trials within switch blocks (i.e., local switch costs, $F(1$, $48)=74.66, p<.001)$. There was also a main effect of time of test and trial type on the Flanker task, $F(2,102)=4.81, p<.05$ and $F(1,51)=358.96 .^{2}$ However, there was also an

\footnotetext{
${ }^{1}$ Parallel analyses were completed on accuracy during the set-switching task and revealed a main effect of trial type, $F(2,96)=$ $42514.85, p<.001$. Follow-up contrasts indicated that both global, $F(1,48)=43013.12, p<.001$, and local, $F(1,48)=61.50, p<.001$, switch costs were present in accuracy.
} 
interaction between time of test and trial type on the Flanker task, $F(2,102)=4.81, p<.05$. Follow-up Helmert contrasts indicated that flanker interference improved from baseline to mid- and post-testing, $F(1,51)=5.29, p<.05$, but did not improve from mid- to post-testing, $F(1,51)=3.21, p>.05$. More importantly, for the present purposes, an interaction between time of test and condition was noted for performance on the letter-number sequencing task, $F(2,49)=3.87, p<.05$. Figure 4 illustrates this interaction. As you can see in this figure, performance on this task improved only in the EC condition. Note also that performance on this task did not differ at baseline as a function of condition. This analysis was repeated using baseline aiming performance as a covariate. The results did not change.

\section{Neuropsychological and Computerized Cognitive Test Battery}

Repeated measures analyses for the additional outcome variables on the neuropsychological test battery are summarized in Table 4 . None of the measures showed a significant interaction between time of test and condition. Means and standard deviations for additional outcome variables are shown in Table 5.

\section{Three-month Follow-up}

This repeated measures analysis included all test sessions, including the assessment that occurred 3 months after the end of training. None of the measures showed a significant interaction between time of test and condition.

\section{DISCUSSION}

This pilot intervention study was designed to investigate the feasibility of using the space fortress game as an intervention modality in elders with the goal of enhancing executive control processes. We selected the SF game because it lends itself to training programs that extend over weeks and months and can be customized to test the efficiency of different training approaches. We selected the SF game with the EC training approach as an intervention modality in older adults because younger adults had shown improved performance on executive controls task following space fortress game training with the EC approach (Fabiani et al., 1989; Gopher, 2007; Gopher et al., 1989, 1994) - and because prior studies suggest that cognitive reserve and executive control are strongly associated. We also selected the EC training approach because older adults show transfer of training on a dual task following EC training with another dual task (Kramer et al., 1995). Finally, we selected the SF game and the EC training approach as an intervention modality in older adults because younger adults show transfer of training to real-world activities such as flight simulation and flying performance (Gopher et al., 1994).

We found that elders were willing and able to play the game, and showed considerable improvement in SF performance over time, regardless of whether they were trained with standard game play instructions or EC instructions. More importantly, for the present purposes, we found that one of our measures of executive control, letter-number sequencing, showed improvement in performance from pre- to post-testing in the EC condition but not in the other two (AC or PC) conditions. This finding is consistent with the suggestion that EC training instructions enhance executive control demands (Arthur et al., 1995; Gopher, 2006; Gopher et al., 1994) and show transfer to training to other cognitive tasks (Kramer et al., 1995). However, unlike computer-based training with the off-the-shelf

\footnotetext{
${ }^{2}$ Parallel analyses were completed on accuracy during the flanker interference task and revealed a main effect of trial type, $F(1,51)=$ $66.38, p<.001$, and an interaction between time of test and trial type, $F(2,102)=3.22, p<.05$. Follow-up contrast indicated that flanker interference improved in the accuracy measure from baseline to mid- and post-testing, $F(1,51)=4.47, p<.05$, but not from mid- to post-testing, $F(1,51)=1.95 p>.05$.
} 
game Rise of Nations (Basak et al., 2008) which showed transfer of learning to a range of different executive control task, including a set-switching task, an N-back task, a visual short-term memory task, and Raven's progressive matrices (Raven, 1990), this was an isolated finding: An SF game play benefit was not noted on the other selected measures of executive control, or on the remainder of the assessment battery. In addition, the benefit was not sustained after 3 months of no game play. Given the limited sample size and power of this pilot study, coupled with multiple statistical comparisons of six primary outcome measures and additional outcome measures, future studies are needed to determine if this isolated finding is indeed reliable. Then again, given that many cognitive interventions developed for cognitively healthy older adults fail to observe transfer of learning (Green \& Bavelier, 2008; Hertzog et al., 2008; Lustig et al., 2009; Noack et al., 2009; Papp et al., 2009; Schmiedek et al., 2010); this isolated finding is noteworthy and potentially informative for the further development of this, and other cognitive interventions aimed to compensate for age-related cognitive decline. In our discussion later, we discuss issues related to the further development of this space fortress intervention in particular and issues related to the further development of cognitive interventions in general.

We specifically hypothesized that SF training with the EC approach would yield the most benefit in our elderly participants. In that respect, the AC condition served as a very strict control condition, where all aspects of game play remain the same except for the specific EC training instructions. Analysis of game play data suggests that the AC and EC conditions resulted in qualitatively different experiences. Contrary to previous experience in younger adults, participants in the AC condition showed greater improvement in total SF performance over the training period. However, this was in great part due to their taking advantage of the bonus score component of the game. While the bonus scores certainly allow participants to follow our instructions, to attempt to achieve the highest scores possible, collecting bonus points is unrelated to the primary mission of the game: to attempt to destroy the space fortress. In addition, collecting bonus points requires no integration of the different aspects of the game; in fact, one could attend to this component of the game without regard to any of the other aspects of game play. In contrast, the participants in the $\mathrm{EC}$ condition were more engrossed in the primary mission of the game. They took more shots at the space fortress, and were more successful in destroying it. It is interesting to note that EC training at no time instructs the participant to focus on destroying the fortress. Rather, the training focuses on ship control and mine handling. However, these two training emphases seem to have induced the participants in the EC condition to immerse themselves in the aspects of game play associated with destroying the space fortress. Because this involved handling the ship, identifying whether mines were friend or foe and destroying them, and attacking the space fortress, we feel it is more likely that EC training induced and guided participants in potential allocation of executive control. It should be noted that these positive aspects of EC training were apparently offset by the additional demands that the EC instructions placed on the elders during their learning of this complex and motorically demanding game. Therefore, the further development of this SF intervention for older adults should consider ways of reducing the motor demands of the SF game - e.g., allowing the player to control the ship with keyboard presses rather than with a joystick.

It is also possible that motor demands are important to consider when developing computerbased cognitive interventions in general. Note that both Rise of Nations and the SF game place a particularly high demand on executive control processes but differ in terms of their motor demands. When playing the SF game, a player has to defend their spaceship against missiles shot by the space fortress, and make decision about friend and foe mines that regularly appear on the screen. When playing Rise of Nations, a player has to defend their city against enemy assault and make decisions about increasing technology and or trade in another city. However, unlike Rise of Nations (which is controlled with keyboard presses 
and mouse clicks); the SF game involves managing a joy-stick controlled space ship that moves in a frictionless environment - a task that involves very precise and discrete motor movements. In other words, it is possible that the Rise of Nation intervention more successfully trained executive control processes than the current SF intervention, because older adults were not also struggling to acquire the fine motor skills involved in controlling the spaceship.

In designing this study, we also recognized the possibility that game play in and of itself would be beneficial regardless of the training approach. For this reason we included a third, no game play control condition. This condition differed radically in that participants were not required to come to the lab three times a week, did not have the regular contact with our study coordinators, and of course had no interaction with any form of a computer game. Thus, while our hypothesis was that EC training would show the greatest cognitive benefit, we also tested whether both forms of game play might enhance performance over the inactive control condition. Our primary finding was that there was differential improvement on a complex working memory task that involves item manipulation (letter-number sequencing) in the EC condition compared to the other two study arms. However, we did not see a condition by time interaction for any of our other selected measures of executive control, or for any of the other measures in our assessment battery. Group size was small, with the resulting limitations and power. Therefore we consider the present findings suggestive at best. However, we do find them encouraging future studies. We also noted a lack of persistence in the benefit of game play after 3 months of no game play. As in other forms of intervention, this suggests that sustained intervention may be needed to produce sustained benefit. In other words, the further development of this and other computer-based cognitive intervention for older adults should consider ways of increasing training time e.g., by allowing older adults to continue game play at home.

It is also possible that the intensity of the training program, and not the hours of training per se, is important to consider when designing computer-based cognitive interventions in general. The current SF pilot intervention involved 21.5 hours of SF game training over the course of 3 months. In contrast, the more successful Rise of Nation intervention (Basak et al., 2008), involved 23.5 hours of game training over the course of 4-5 weeks. In other words, the further development of computer-based cognitive interventions should also consider, and have the potential to customize (or systematically examine), the duration and the frequency of training sessions over the course of the intervention.

Another consideration when examining the potential benefit of this SF intervention is whether our selected neuropsychological and cognitive measures could optimally capture the benefit of game play. In studies of younger adults, SF game play was associated with more successful training on piloting jets or helicopters. These are real world tasks that call on many of the skills enhanced by the game. It is possible that game play enhances aspects of real-world behavior that call for multitasking, attentional allocation, and decision-making, but that our assessment battery was not sensitive to these benefits. More specifically, our primary outcome variables were selected to represent different executive control processes. Letter-number sequencing was selected because it involves item manipulation, and because it has been linked to work attainment and skills in patient populations (Bowie et al., 2008; Murtagh et al., 2010). Trails B-A and switch costs (local and global) were selected because they assess a person's ability to switch between two different task sets. Finally, Stroop Interference and Flanker Interference were selected because they involve response competition or interference (Sanders \& Lamers, 2002). Thus, while we included traditional measures of set shifting, set maintenance, and bottom-up interference our assessment battery did not include a dual task situation. Given that EC instructions during SF game learning encourage participants to shift their attention from one task (ship handling) to another task 
(mine handling), and older adults trained with EC instructions in one dual-task situation show transfer of training to another dual task situation (Kramer et al., 1995), it is possible that a dual task would have been more sensitive to transfer of training following SF game learning as well. Thus, although this pilot study might suggest a potential benefit of space fortress training, the problem of developing outcome measures that are sensitive to these benefits must be addressed more carefully.

\section{CONCLUDING COMMENTS}

The current study investigated the feasibility of using the SF game with the EC training approach to improve executive control processes in cognitively healthy older adults. We found limited transfer of training to one measure of executive control, the letter-number sequencing task, from pre- to post-evaluations in the EC training condition, but not in the other two training ( $\mathrm{AC}$ or $\mathrm{PC}$ ) conditions. These preliminary findings are limited but provide important information regarding the further development of this SF intervention in particular and computer-based cognitive interventions in general. More specifically, future computerbased cognitive interventions should consider the motor demands of the game, the duration and the intensity of the intervention, providing at-home game training, and selecting appropriate outcome measures. Finally, the further development of computer-based cognitive interventions should consider that a combination of cognitive training and aerobic exercise may generate greater transfer of training effects (Colcombe \& Kramer, 2003). A combination of cognitive training and aerobic exercise may generate greater transfer effects because aerobic exercise alone has been shown to improve executive control processes in humans (Kramer et al., 1999), and because aerobic exercise has been shown to promote an increase in neurogenesis (van Praag, Kemperman, \& Gage, 1999).

\section{Acknowledgments}

We would like to thank the Taub Foundation for providing support for this research. This research was also supported by T32AG00261.

\section{REFERENCES}

Arthur W Jr, Strong MH, Jordan JA, Williamson JE, Shebilske WL, Regian JW. Visual attention: Individual different in training and predicting complex task performance. Acta Psychologica. 1995; $88: 3-23$.

Basak C, Boot WR, Voss MW, Kramer AF. Can training in a real-time strategy video game attenuate cognitive decline in older adults? Psychology and Aging. 2008; 23:765-777. [PubMed: 19140648]

Benton, AL.; Hamsher, KD. Multilingual aphasia examination. University of Iowa; Iowa City, IA: 1976.

Bherer L, Kramer AF, Peterson MS, Colcombe S, Erickson K, Becic E. Training effects on dual task performance: Are there age-related differences in plasticity of attentional control? Psychology and Aging. 2005; 20:695-709. [PubMed: 16420143]

Bherer L, Kramer AF, Peterson MS, Colcombe S, Erickson K, Becic E. Transfer effects in task-set cost and dual-task cost after dual-task training in older and younger adults: Further evidence for cognitive plasticity in attentional control in late adulthood? Experimental Aging Research. 2008; 34:188-219. [PubMed: 18568979]

Blumen HM, Gopher D, Steinerman JR, Stern Y. Training cognitive control in older adults with the Space Fortress game: The role of training instructions and basic motor ability. Frontiers in Aging Neuroscience. 2010; 2(145):1-12. [PubMed: 20552041]

Bowie CR, Leung WW, Reichenberg A, McClure MM, Patterson TL, Heaton RK, Harvey PD. Predicting schizophrenia patients' real-world behavior with specific neuropsychological and functional capacity measures. Biological Psychiatry. 2008; 63(5):505-511. [PubMed: 17662256] 
Colcombe S, Kramer AF. Fitness effects on the cognitive function of older adults: A meta-analytic study. Psychological Science. 2003; 14:125-130. [PubMed: 12661673]

Drew B, Waters J. Video games: Utilization of a novel strategy to improve perceptual motor skills and cognitive functioning in the non-institutionalized elderly. Cognitive Rehabilitation. 1986; 4:26-31.

Delis, DC.; Kramer, JH.; Kaplan, E.; Ober, BA. California Verbal Learning Test (CVLT) - Second Edition. The Psychological Corporation; San Antonio, TX: 2000.

Fabiani M, Buckley J, Gratton G, Coles MGH, Donchin M, Logie R. The training of complex task performance. Acta Psychologica. 1989; 71:259-299.

Frederiksen JR, White BY. An approach to training based upon principled task decomposition. Acta Psychologica. 1989; 71:89-146.

Golden, CJ. Stroop Color and Word Test. Stolting; Chicago, IL: 1978.

Goodglass, H.; Kaplan, E. The assessment of aphasia and related disorders. Lea \& Febiger; Philadelphia, PA: 1983.

Gopher, D. Control processes in the formation of task units. In: Qichen, J., editor. Psychological science around the world, Vol. 2, Social and applied issues. Oxford Psychology Press; Hove, UK: 2006. p. 385-404.A chapter based on a keynote address given at the 28th International Congress of Psychology

Gopher, D. Emphasis change as a training protocol for high demands tasks. In: Kramer, A.; Wiegman, D.; Kirlik, A., editors. Attention from theory to practice. Oxford Psychology Press; Oxford, UK: 2007.

Gopher D, Weil M, Siegel D. Practice under changing priorities: An approach to training of complex skills. Acta Psychologica. 1989; 71:147-178.

Gopher D, Weil M, Bareket T. Transfer of skill from a computer game trainer to flight. Human Factors. 1994; 36:387-405.

Green CS, Bavelier D. Action video game modifies visual selective attention. Nature. 2003; 423:534537. [PubMed: 12774121]

Green CS, Bavelier D. Effect of action video games on the spatial distribution of visuospatial attention. Journal of Experimental Psychology: Human Perception \& Performance. 2006; 32:1465-1478. [PubMed: 17154785]

Green CS, Bavelier D. Action-video-game experience alters the spatial resolution of vision. Psychological Science. 2007; 18:88-94. [PubMed: 17362383]

Green CS, Bavelier D. Exercising your brain: A review of human brain plasticity and training-induced learning. Psychology and Aging. 2008; 23:692-701. [PubMed: 19140641]

Hertzog C, Kramer AF, Wilson RS, Lindenberger U. Enrichment effects on adult cognitive development: Can the functional capacity of older adults be preserved and enhanced? Psychological Science in the Public Interest. 2008; 9:1-65.

Karbach J, Kray J. How useful is executive control training? Age differences in near and far transfer of task-switching training. Developmental Science. 2009; 12:978-990. [PubMed: 19840052]

Kramer AF, Larish JF, Strayer DL. Training attentional control in dual-task settings: A comparison of young and old adults. Journal of Experimental Psychology: Applied. 1995; 1:50-76.

Kramer AF, Hahn S, Cohen NJ, Banich MT, McAuley E, Harrison CR, Chason J, Vakil E, Bardell L, Boileau RA, Colcombe A. Ageing, fitness and neurocognitive function. Nature. 1999; 400:418419. [PubMed: 10440369]

Lustig C, Shah P, Seidler R, Reuter-Lorenz PA. Aging, training and the brain: A review and future directions. Neuropsychology Review. 2009; 19:504-522. [PubMed: 19876740]

Mané AM, Donchin E. The Space Fortress game. Acta Psychologica. 1989; 71:17-22.

Mattis, S. Mental status examination for organic mental syndrome in the elderly patient. In: Bellak, L.; Karasu, TB., editors. Geriatric psychiatry. Grune \& Stratton; New York, NY: 1976. p. 77-121.

Murtagh A, Hurley AL, Kinsella A, Corvin A, Donohoe G, Gill M, O’Callaghan E, Murphy KC. The letter number sequencing test and its association with potential to work among people with psychotic illness. European Psychiatry. 2010; 25:101-104. [PubMed: 19720503] 
Norman, DA.; Shallice, T. Attention to action: Willed and automatic control of behavior. In: Davidson, RJ.; Schwartz, GE.; Shapiro, D., editors. Consciousness and self-regulation. Vol. 4. Plenum; New York, NY: 1986. p. 376-390.

Noack H, Lövdén M, Schmiedek F, Lindenberger U. Cognitive plasticity in adulthood and old age: Gauging the generality of cognitive intervention effects. Restorative Neurology \& Neuroscience. 2009; 27:435-453. [PubMed: 19847069]

Papp KV, Walsh SJ, Snyder PJ. Immediate and delayed effects of cognitive interventions in healthy elderly: A review of current literature and future directions. Alzheimer's \& Dementia. 2009; 5:5060.

Reimers S, Maylor EA. Task switching across the lifespan: Effects of age on general and specific switch costs. Developmental Psychology. 2005; 41:661-671. [PubMed: 16060812]

Reitan, R. Manual for Administration of Neuropsychological Test Batteries for Adults and Children. Reitan Neuropsychology Laboratories; Tucson, AZ: 1978.

Rebok GW, Carlson MC, Langbaurn JBS. Training and maintaining memory abilities in healthy older adults: Traditional and novel approaches. Journals of Gerontology Series B - Psychological Sciences and Social Sciences. 2007; 62:53-61.

Raven, JC. Advanced Progressive Matrices. Set II. Oxford Psychology Press; Oxford, UK: 1990.

Sanders AF, Lamers JM. The Eriksen flanker effect revisited. Acta Psychologica. 2002; 109:41-56. [PubMed: 11766139]

Schmiedek F, Bauer C, Lövdén M, Bose A, Lindenberger U. Cognitive enrichment in older age. GeroPsych: The Journal of Gerontopsychology and Geriatric Psychiatry. 2010; 23:59-67.

Smith S, Jenkinson M, Beckmann C, Miller K, Woolrich M. Meaningful design and contrast estimability in FMRI. Neurolmage. 2007; 34:127-136.

Shebilske WN, Volz RA, Gildea KM, Workman JW, Nanjanath M, Cao S, Whetzel J. Revised Space Fortress: A validation study. Behavior Research Methods. 2005; 37:591-601. [PubMed: 16629291]

Siedlecki KL, Stern Y, Reuben A, Sacco RL, Elkind MSV, Wright CB. Construct validity of cognitive reserve in a multiethnic cohort: The Northern Manhattan Study. Journal of the International Neuropsychological Society. 2009; 15:558-569. [PubMed: 19573274]

Stern Y. Cognitive reserve. Neuropsychologia. 2009; 47:2015-2028. [PubMed: 19467352]

Stern Y, Zarahn E, Habeck C, Holtzer R, Rakitin BC, Kumar A, Flynn J, Steffener J, Brown T. A common neural network for cognitive reserve in verbal and object working memory in young but not old. Cerebral Cortex. 2008; 18:959-967. [PubMed: 17675368]

van Praag H, Kempermann G, Gage FH. Running increases cell proliferation and neurogenesis in the adult mouse dentate gyrus. Nature Neuroscience. 1999; 2:266-270.

Verhaeghen P, Marcoen A, Goossens L. Improving memory performance in the aged through mnemonic training: A meta-analytic study. Psychology and Aging. 1992; 7:242-251. [PubMed: 1535198]

Wechsler, D. Wechsler Adult Intelligence Scale - III. Psychological Corporation; San Antonio, TX: 1997.

Willis SL, Tennstedt SL, Marsiske M, Ball K, Elias J, Koepke KM, Morris JN, Rebok GW, Unverzagt FW, Stoddard AM, Wright E. Long-term effects of cognitive training on everyday functional outcomes in older adults. The Journal of the American Medical Association. 2006; 296:28052814. 


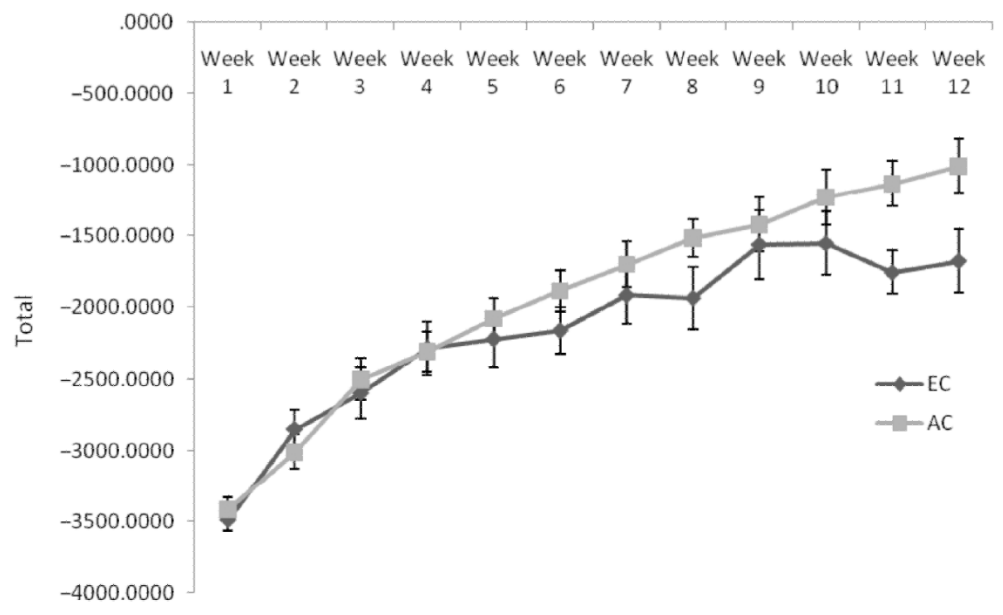

FIGURE 1.

Total SF performance as a function of training instruction (EC vs. AC), adjusted by subjectspecific aiming scores. 


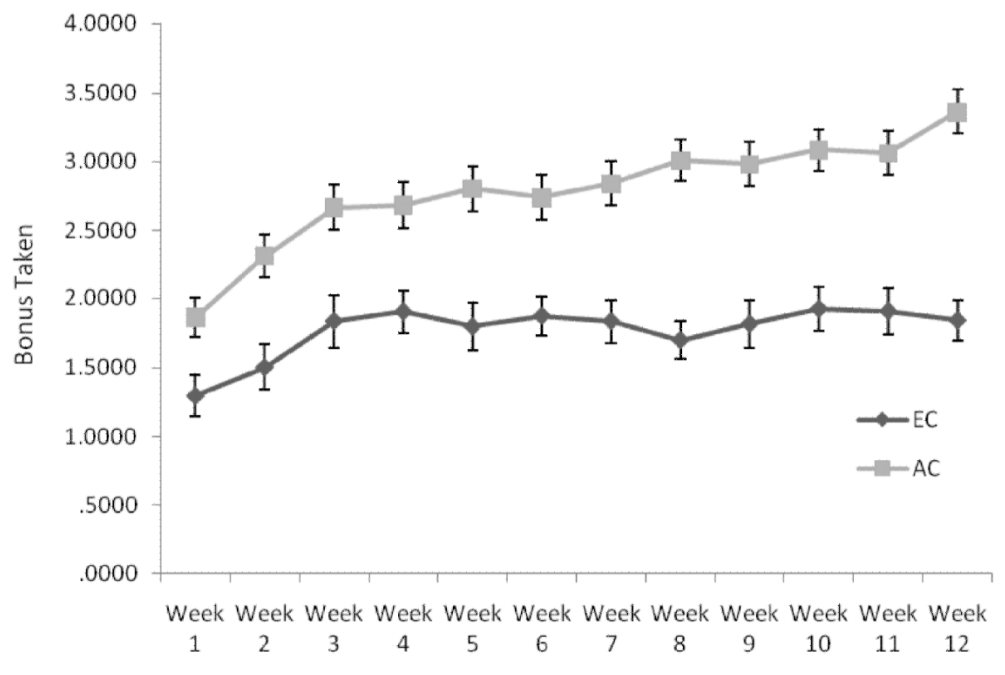

FIGURE 2.

Bonus Taken as a function of training instructions (EC vs. AC), adjusted by subject-specific aiming scores. 


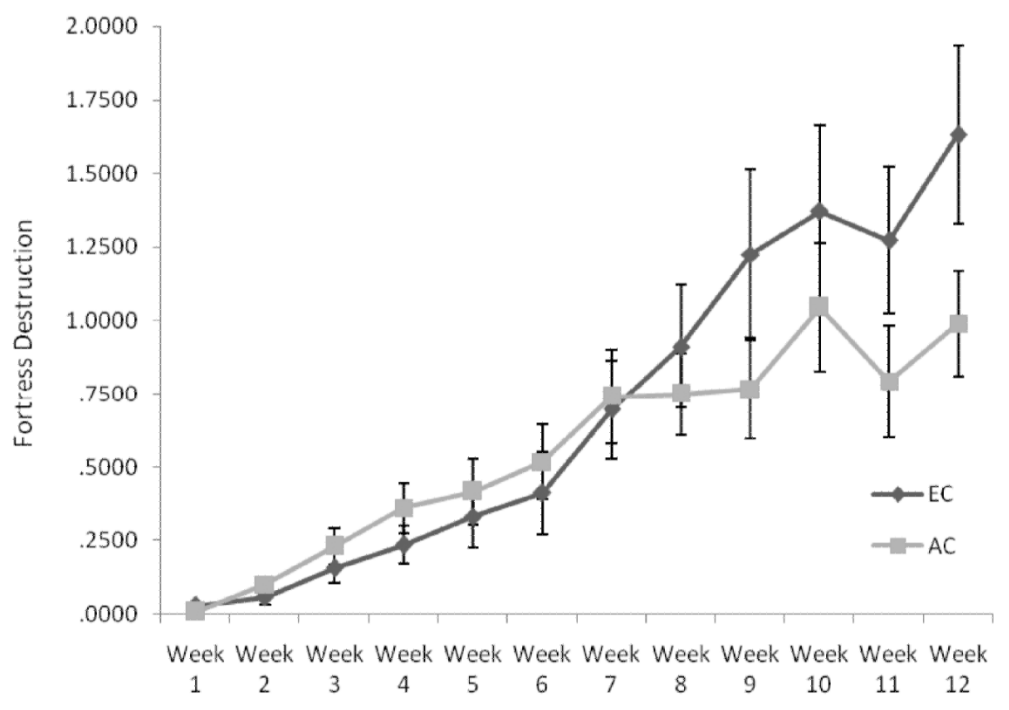

FIGURE 3.

Fortress Destructions as a function of training instructions (EC vs. AC) in participants with high (>450) subject-specific aiming scores. 


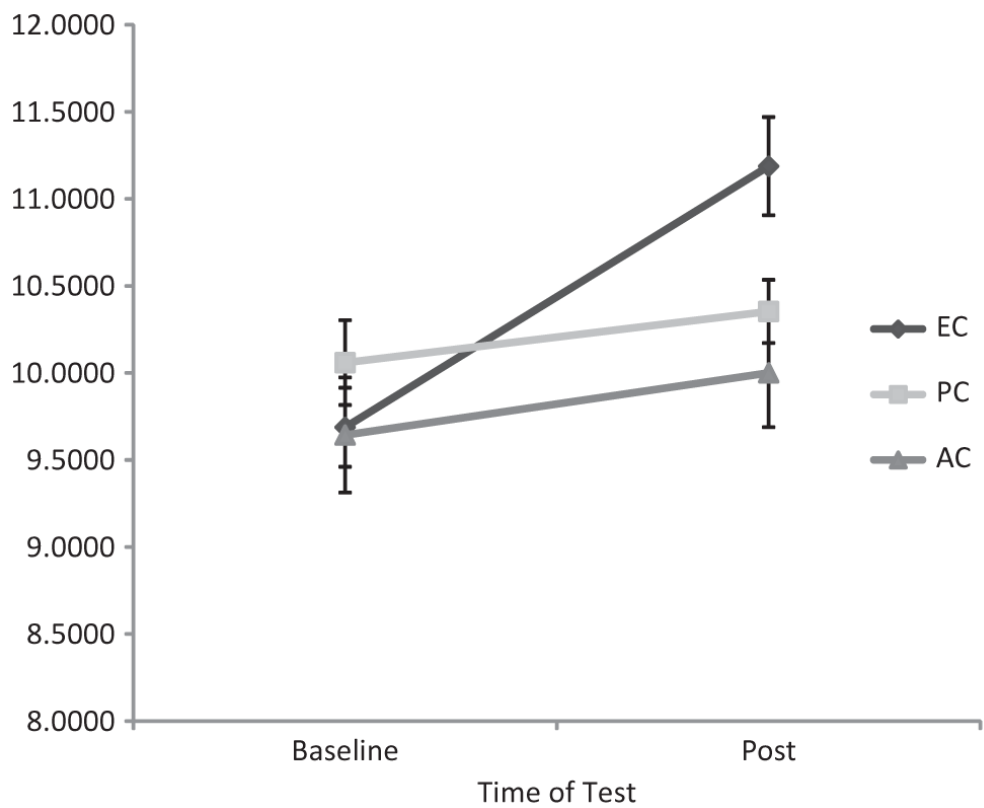

FIGURE 4.

Letter number sequencing in the $\mathrm{EC}, \mathrm{AC}$ and $\mathrm{PC}$ condition at baseline and post-game training. 


\section{TABLE 1}

Means and standard deviations (in parentheses) of demographic and screening information for participants in the emphasis change (EC) condition, the active control (AC) condition, and the passive control (PC) condition

\begin{tabular}{|llll|}
\hline Condition & EC & AC & PC \\
\hline Age & $66.35(4.55)$ & $65.56(4.05)$ & $66.95(4.16)$ \\
\% Female & 0.59 & 0.50 & 0.58 \\
DRS (Total) & $140.71(2.76)$ & $140.67(2.33)$ & $140.21(2.76)$ \\
Years of education & $15.65(2.55)$ & $16.28(2.61)$ & $15.74(2.54)$ \\
WAIS vocabulary (raw) & $55.47(7.74)$ & $54.38(12.46)$ & $55.06(11.20)$ \\
WTAR (raw) & $41.00(8.63)$ & $42.17(7.49)$ & $40.16(9.53)$ \\
\hline
\end{tabular}




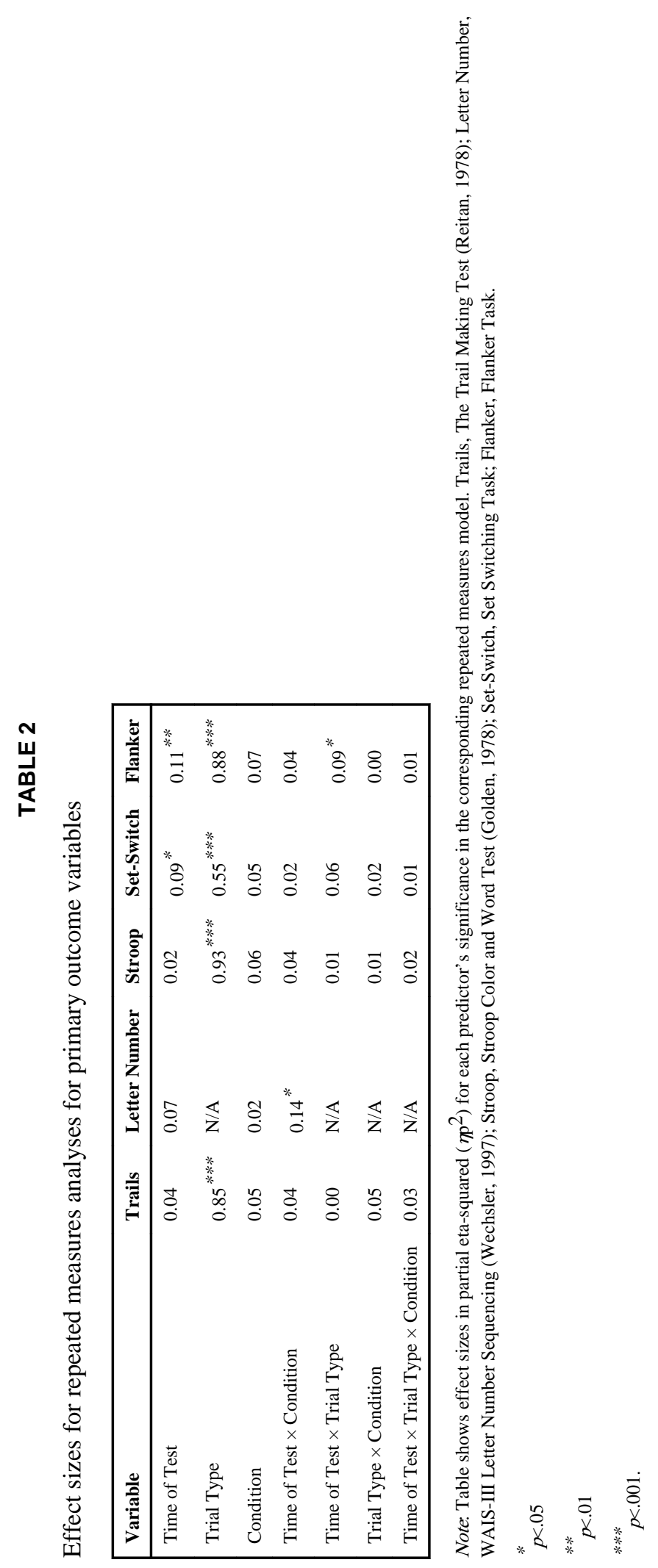

Neuropsychol Dev Cogn B Aging Neuropsychol Cogn. Author manuscript; available in PMC 2013 March 07. 


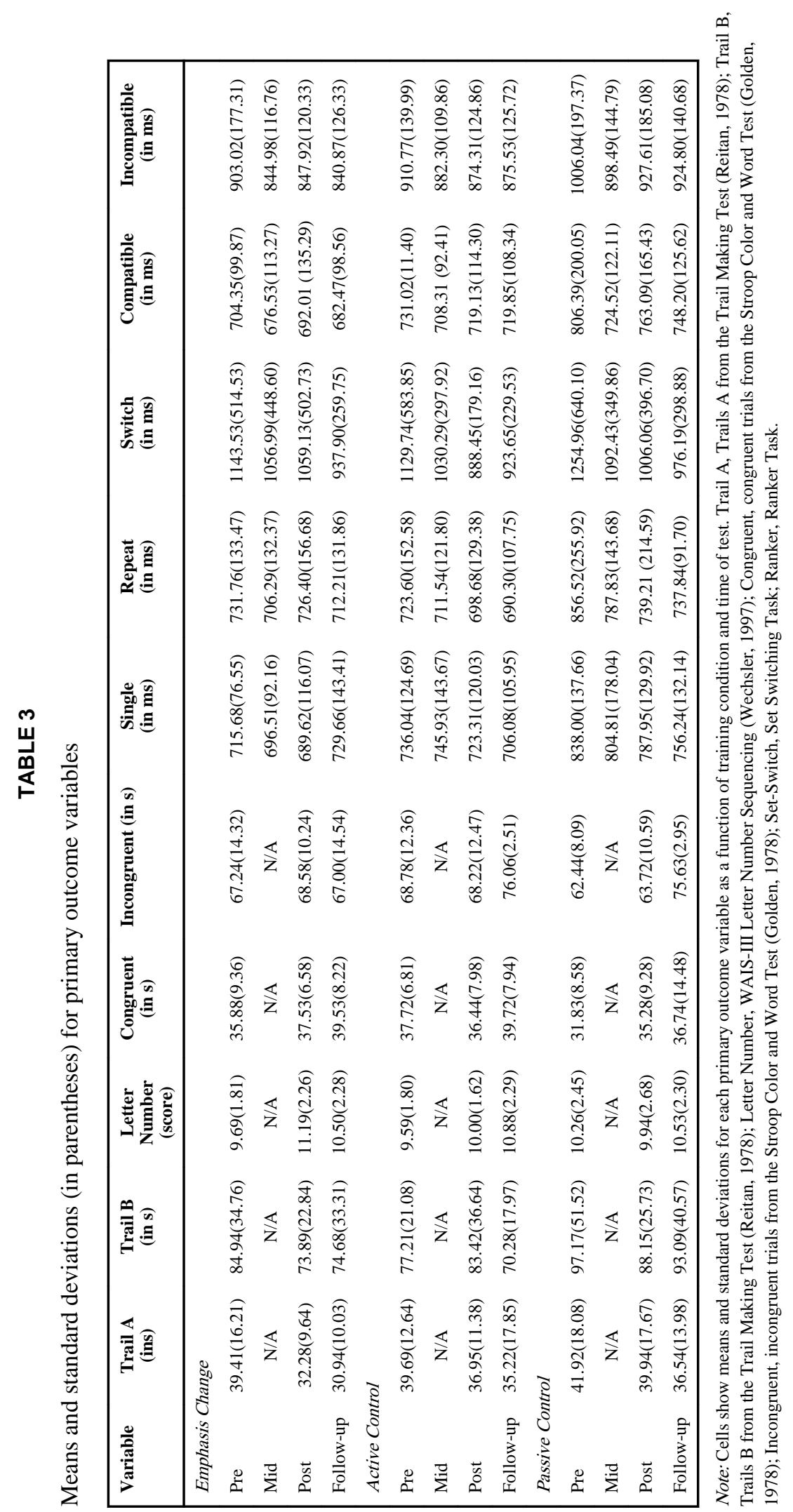




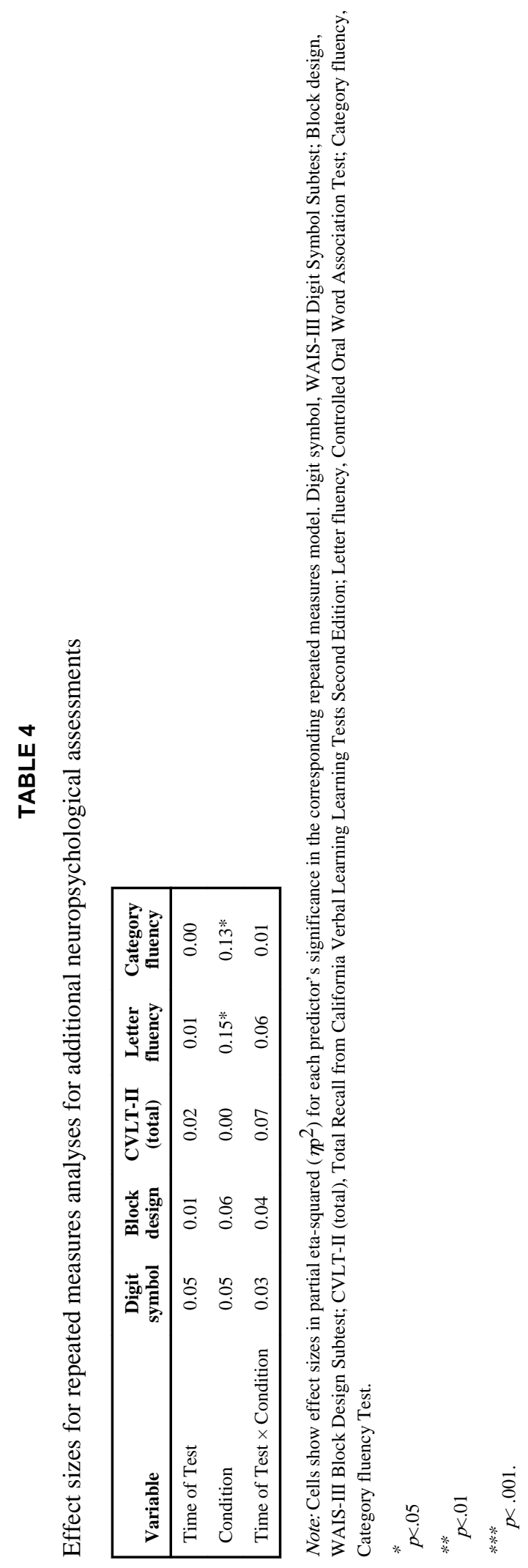

Neuropsychol Dev Cogn B Aging Neuropsychol Cogn. Author manuscript; available in PMC 2013 March 07. 


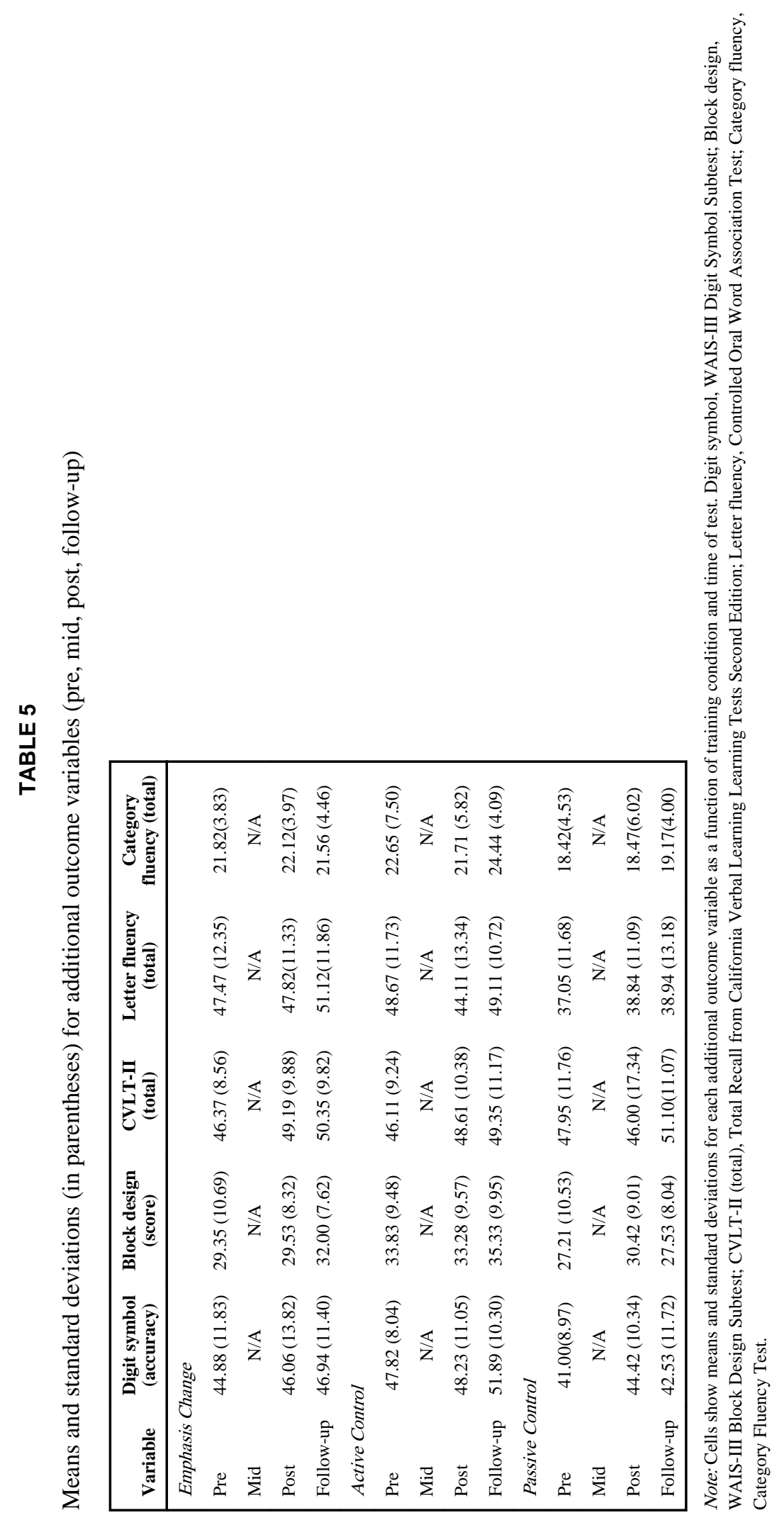

Neuropsychol Dev Cogn B Aging Neuropsychol Cogn. Author manuscript; available in PMC 2013 March 07. 